\title{
Convection and Chemistry Effects in CVD- A 3-D Analysis for Silicon Deposition
}

S.A. Gökoğlu

Case Western Reserve University

Cleveland, Ohio

M.A. Kứzmarski

National Aeronautics and Space Administration

Lewis Research Center

Cleveland, Ohio

P. Tsui

Sverdrup Technology, Inc.

NASA Lewis Research Center Group

Cleveland, Ohio

and

A. Chait

National Aeronautics and Space Administration

Lewis Research Center

Cleveland, Ohio

Prepared for the

Euro CVD 7. Conference

sponsored by the Institut de Science et de Genie des Materiaux et Procedes

Perpignan, France, June 19-23, 1989 


\title{
CONVECTION AND CHEMISTRY EFFECTS IN CVD - A 3-D
}

\section{ANALYSIS FOR SILICON DEPOSITION}

\author{
S.A. Gökoğlu* \\ Case Western Reserve University \\ Materials Science and Engineering Department \\ Cleveland, Ohio 44106 \\ M.A. Kuczmarski \\ National Aeronautics and Space Administration \\ Lewis Research Center \\ Cleveland, Ohio 44135 \\ P. Tsui \\ Sverdrup Technology, Inc. \\ NASA Lewis Research Center Group \\ Cleveland, Ohio 44135 \\ and \\ A. Chait \\ National Aeronautics and Space Administration \\ Lewis Research Center \\ Cleveland, Ohio 44135
}

\begin{abstract}
The computational fluid dynamics code FLUENT has been adopted to simulate the entire rectangular-channel-like (3-D) geometry of an experimental CVD reactor designed for Si deposition. The code incorporated the effects of both homogeneous (gas phase) and heterogeneous (surface) chemistry with finite reaction rates of important species existing in silane dissociation. The experiments were designed to elucidate the effects of gravitationally-induced buoyancydriven convection flows on the quality of the grown si films. This goal is accomplished by contrasting the results obtained from a carrier gas mixture of $\mathrm{H}_{2} / \mathrm{Ar}$ with the ones obtained from the same molar mixture ratio of $\mathrm{H}_{2} / \mathrm{He}$, without any accompanying change in the chemistry. Computationaliy, these"cases are simulated in" the terrestrial gravitational field and in the absence of gravity. The numerical results compare favorabiy with experiments." Powerful computational tools provide invaluable insights into the complex physicochemical phenomena taking place in CVD reactors. Such information is essential for the improved design and optimization of future CVD reactors.
\end{abstract}

\section{1 - INTRODUCTION}

Despite its routine application for the production of many electronic, optical and structural materials, the level of understanding in CVD processes has been rather limited. This is evident from the conventional approaches, taken by CVD reactor designers, which are mostly based on intuition and experience. The sometimes unjustified assumptions and simplifications employed by many modellers are also consequences of insufficient fundamental knowledge about the complex physicochemical interactions involved. Extensive discussions of available CVD models are given in the recent reviews of Hess, Jensen and Anderson/I/ and Jensen/2/. Indeed, a comprehensive analysis should simultaneously include, among other things, homogeneous(gas phase) and

*NASA Senior Resident Research Associate. 
heterogeneous (surface) chemical reaction kinetics, heat and multicomponent mass transport, fluid physics and thermodynamics. In many cases, these phenomena should be treated in multidimensions, and, in some, even the 2-D approaches fail to provide the necessary insight, as is demonstrated by Rosenberger $/ 3 /$.

There are, however, some reactor/flow/susceptor configurations and thermal conditions where certain approximations can be made. For such cases, an extra level of sophistication is not warranted. Stagnation flow reactors studied by Rebenne and Pollard/4/, Houfman, Graves and Jensen/5/ and Wahl,et al./6/, or rotating disk reactors studied by Evans and Grief/7/ and Coltrin et al./8/, or horizontal reactors studied by Coltrin, Kee and Miller/9,10/ and Ristorcelli and Mahajan/11/ are examples where the analyses are justifiably reduced to 2-I with negligible sacrifice in information.

Recently, increasing interest in the "CAD-CAM" approach to reactor design methodologies and improving availability and quality of homogeneous chemical kinetic data have to motivated full 3-D transport analyses. The application of the computational package PHOENICS by Rhee, Szekely and Ilegbusi/12/ to the analysis of a rectangular reactor, neglecting both homogeneous and

heterogeneous finite rate chemistry, and the systematic study of transport phenomena in horizontal and tilted CVD reactors by Moffat and Jensen/13/ demonstrate such trends.

Presently, the computational fluid dynamics code FLUENT is being enhanced and extended under a NASA program to incorporate many of the phenomena encountered in CVD. The intermediate results of this development effort are reported in references 14 and 15 . We have adopted this intermediate version of FLUENT-CVD to study the effects of both buoyancy and chemistry in the si deposition reactor designed by Tsui/16/ (see also Tsui, P. and Spear, K.E., to be published).

The nearly two-decade old $\mathrm{Si}$ deposition rate results of Eversteyn et al./17/ have so far been the only experimental data for modellers to test their codes against. Relevant and reliable CVD rate data is hardly available in the literature for the validation of sophisticated models under stringent conditions. Tsui's/16/ Si deposition experiments were, however, designed to address a modeller's needs. Therefore, they provide excellent benchmark cases for code verification.

Tsui's experiments were designed to elucidate the the role of gravitationally-induced buoyancy-driven convection flows on the CVD rates and the quality of the Si films grown. Thus, rates obtained from different carrier gas mixtures of $\mathrm{H}_{2} / \mathrm{He}$ are compared with the ones obtained from corresponding molar mixtures of $\mathrm{H}_{2} / \mathrm{Ar}$. This assures that no accompanying change in the chemistry is introduced. The experiments were performed at various substrate temperatures using 0.1 mole percent silane as the Si-source gas. Previous modelling efforts of stinespring and Annen/18/ for these experiments predicted qualitative and quantitative trends which did not agree with the experimental observations. This can be mainly attributed to a) their model assumes fully developed velocity and temperature fields at the hot substrate leading edge with no entrance length for their development, b) their species equations are only 2-D in a 3-D flow field, c) their computational volume takes the side edges of the susceptor as the location of reactor side wall which oversimplifies the real situation (indeed, this assumption is routinely employed by even the 3-D modellers in the literature with sometimes serious consequences, i.e. different temperature fields, aspect ratios, buoyancy effects, etc., and is not a realistic description of typical CVD reactors), and d) they assume thermochemical equilibrium (infinitely fast reaction rates) in the gas phase.

The main objective of this study is to address the question of how significant the effect of buoyancy is on the quality of the crystals grown by CVD. Our preliminary comparison with experiments indicate that FLUENT-CVD can correctly describe many of the $3-D$ physicochemical phenomena occuring in a typical CVD reactor. However, more rigorous tests are needed to confidently validate the code. The results of such a thorough comparison will be reported elsewhere (Gokoglu et al., to be published). 
The details of the transport equations used by FLUENT-CVD describing the conservation of mass, momentum(Navier-Stokes), energy and species mass are given in references 19 and 20. In Cartesian tensor notation they can be written as :

Total Mass :

$\partial / \partial x_{i}\left(\rho u_{i}\right)=0$

Momentum :

$\partial / \partial x_{\ell}\left(\rho u_{i} u_{\ell}\right)=-\partial p / \partial x_{i}+\partial / \partial x_{\ell}\left[\mu\left(\partial u_{i} / \partial x_{\ell}+\partial u_{\ell} / \partial x_{i}\right)\right]+\rho g_{i}$

Energy :

$\partial / \partial x_{i}\left(\rho u_{i} h\right)=\partial / \partial x_{i}\left(\lambda / c_{p} \cdot \partial h / \partial x_{i}\right)$

Species Mass :

$\partial / \partial x_{i}\left(\rho u_{i} Y_{j}\right)=\partial / \partial x_{i}\left(\mu / S c_{j} \cdot \partial Y_{j} / \partial x_{j}\right)+\Sigma_{k=1}^{K} R_{j k}$

The temperature, $T$, is obtained from the enthalpy, $h$, via the relationship $\mathrm{dh}=c_{\mathrm{p}} \mathrm{dT}$. The mixture density, $\rho$, is calculated from the ideal gas law using the equation :

$\rho=p /\left(\operatorname{RT} \Sigma_{j=1}^{J} Y_{j} / W_{j}\right)$

where $J$ is the total number of gaseous species. The dynamic viscosity, $\mu$, and the thermal conductivity, $\lambda$, of the mixture are obtained from the semiempirical formula of Wilke/21/. The mixture specific heat, $c_{p}$, is calculated from the molar weighted sum of the individual species specific heats. Polynomial curve fits in $T$ are subsequently calculated to describe the temperature dependences for each of $\mu, \lambda$ and $c_{p}$ to be eventually used by FLUENT-CVD. Natural convection is included via the gravitational body force in Eq. (2) and the temperature dependence of $\rho$ given by Eq.(5). The Schmidt number, $\mu /\left(\rho D_{j}\right)$, is assumed to be constant for each species. Therefore, the diffusion coefficient, $D_{j}$, will have the same temperature dependence as $\mu / \rho$.

It should be noted that currently Eq. (4) does not include thermal(Soret) diffusion of species. This is certainly not a justifiable assumption for most CVD applications where steep temperature gradients exist/22,23/. Further development work of FLUENT-CVD includes plans to incorporate thermal diffusion in the near future.

It should also be noted that because the si-carrier species are dilute, $\left(\mathrm{Y}_{j} \ll 1\right.$ ), in the host gas mixture, a number of simplifications can be made, such as 1 ) neglecting the effect of si-carrier species compositions on fluid properties, $\mu, \lambda$ and $c_{p}$, and the species diffusion coefficient, $\left.D_{j}, 2\right)$ using the binary, rather than the multicomponent, diffusion coefficients for each species in the mixture, 3) neglecting the rate of creation of energy by homogeneous chemical reactions in Eq. (3) and by surface reactions, and 4) neglecting the energy transport associated with species mass fluxes (Dufour effect) in Eq. (3).

\section{Homogeneous Reactions :}

Rather than employing the complex system of reactions for si deposition from silane dissociation involving more than 20 different molecules, as reported by Coltrin, Kee and Miller/9,10\%, we have limited the significant gas phase molecules to silane, silylene and disilane, based on the discussion given by Moffat and Jensen $13 \%$. Indeed, since there is no experimental evidence to suggest otherwise, this approach is justified in hydrogen rich environments, as is the case studied here. Therefore, the reduced reactions are given by: 


$$
\begin{aligned}
& \mathrm{SiH}_{4} \underset{\mathrm{k}_{1, \mathrm{r}}}{\mathrm{k}_{1}, \mathrm{f}} \mathrm{SiH}_{2}+\mathrm{H}_{2}
\end{aligned}
$$

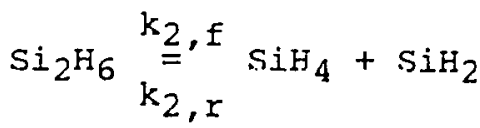

An Arrhenius type rate expression, $k=A T^{\beta} \exp (-E / R T)$, is used for the forward and reverse reaction rate constants, given by/13/

\begin{tabular}{|c|c|c|c|c|c|}
\hline Reaction & $\begin{array}{l}\text { Molar } \\
\text { Mixture }\end{array}$ & $A\left(s^{-1}\right)$ & $\mathrm{A}\left(\mathrm{m}^{3} / \mathrm{kg}-\mathrm{s}\right)$ & $\beta$ & $E(\mathrm{~J} / \mathrm{kgmol})$ \\
\hline $\begin{array}{l}1, f \\
2, f \\
1, r \\
1, r \\
2, r \\
2, r\end{array}$ & $\begin{array}{c}\text { all } \\
\text { all } \\
25 \% \mathrm{He} / 75 \% \mathrm{H}_{2} \\
25 \% \mathrm{Ar} / 75 \% \mathrm{H}_{2} \\
25 \% \mathrm{He} / 75 \% \mathrm{H}_{2} \\
25 \% \mathrm{Ar} / 75 \% \mathrm{H}_{2}\end{array}$ & $\begin{array}{c}6.10 \cdot 10^{28} \\
2.12 \cdot 10^{35} \\
-- \\
-- \\
-- \\
--\end{array}$ & $\begin{array}{c}-- \\
-- \\
2.087 \cdot 10^{21} \\
4.583 \cdot 10^{20} \\
7.075 \cdot 10^{23} \\
1.554 \cdot 10^{23}\end{array}$ & $\begin{array}{l}-5.00 \\
-6.47 \\
-4.44 \\
-4.44 \\
-4.50 \\
-4.50\end{array}$ & $\begin{array}{l}2.4614 \cdot 10^{8} \\
2.3597 \cdot 10^{8} \\
1.4267 \cdot 107 \\
1.4267 \cdot 107 \\
1.2845 \cdot 10^{8} \\
1.2845 \cdot 10^{7}\end{array}$ \\
\hline
\end{tabular}

\section{Modelled Reactor and Boundary Conditions :}

The modelled portion of the schematic overview of Tsui's experimental reactor is given in Figure 1. Full details of the actual reactor are given in reference 16. The susceptor is made of silicon carbide coated graphite and is inductively heated. The substrate is a $5.08 \mathrm{~cm}$ long by $2.54 \mathrm{~cm}$ wide silicon wafer centered on the susceptor. The solid block on which the susceptor is placed and the block upstream of the susceptor are both made of quartz. These solid bodies are naturally heated by heat conduction from the hot susceptor. A surface temperature of $1373 \mathrm{~K}$ is used for the heated susceptor top, side and back surfaces. The reactor unheated (cold) surfaces are assumed to be at $300 \mathrm{~K}$ everywhere except at the bottom quartz surface of the rectangular entrance duct upstream of the heated susceptor. The conjugate heat transfer capabilty of FLUENT-CVD is utilized in that region to actually calculate the steady state equilibrium surface temperatures resulting from heat conduction along the bottom surface of the entrance region prior to the deposition surface.

In order to simulate the experimental conditions, we have used the same $4 \mathrm{slm}$ total carrier gas $\left(75 \% \mathrm{H}_{2}\right.$ with $25 \%$ He or $75 \% \mathrm{H}_{2}$ with $25 \%$ Ar by mole) flowrate with 0.1 mole percent silane at $300 \mathrm{~K}$ for the reactor inlet conditions. At the reactor exit, the program considers global mass conservation and imposes a zero normal gradient condition for the solution variables. On the solid boundaries, the non-slip condition is used for velocity components parallel to the walls. Because the si-carrier reactive species are dilute in the mixture, a zero convective flux condition is used normal to the walis.

As the boundary condition for species on heated surfaces we use the balance between the diffusional mass flux of each species to/from the surface and the local rate of creation/destruction of that species due to chemical reactions at the surface. Thus, for each chemical species, $j$,

$$
-\rho D_{j} n \cdot \nabla Y_{j}=\Sigma_{k=1}^{K} R_{j k}^{S}
$$

at the surface. Note again the absence of the thermal(soret) diffusion term in the species diffusional mass flux expression. Although Eq.(6) applies on all surfaces, for practical computational efficiency purposes a zero species mass flux condition is used on the unheated walls with negligable loss in the accuracy of results. The growth rates of $\mathrm{Si}$ on heated surfaces can then be obtained from the deposition rates by

$$
\text { Growth rate(length/time) }=\left[\rho_{S i(s)}\right]^{-1} \sum_{k=1}^{K} R_{S i(s) k}^{S}
$$


The $j$ th surface reaction rate can be expressed as the product of the molecular flux across the Knudsen sublayer (estimated from the frequency of molecular surface collisions using the kinetic theory) and the reaction probability, $r_{j}$, (sometimes referred to as the sticking coefficient) of the $j$ th species

$$
R_{j}^{S}=\gamma_{j}\left[R T /\left(2 \pi W_{j}\right)\right]^{1 / 2} \rho Y_{j}
$$

Because FLUENT-CVD requires the surface reactions expressed in terms of Arrhenius type $\left[\mathrm{AT}^{\beta} \exp (-\mathrm{E} / \mathrm{RT})\right]$ rate constants (unless they are provided by user supplied subroutines), Eq.(8) is rewritten in the form of a product of a pseudo-first order reaction rate constant and concentration $\left(\rho Y_{j}\right)$ for each species. We have also used the surface reaction probability expression of coltrin, Kee and Miller/10/ for silane given by

$$
r_{S i H 4}=5.37 \cdot 10^{-2} \exp (-9400 / T)
$$

which can be combined with the Arrhenius rate constant expression. The surface reaction probabilities of the other reactive Si-carrier species, $\mathrm{SiH}_{2}$ and $\mathrm{Si}_{2} \mathrm{H}_{6}$, are taken to be unity whereas those of $\mathrm{H}_{2}$ and the inert gases to be

\begin{tabular}{|c|c|c|c|}
\hline Species & $A\left(m / s-K^{1 / 2}\right)$ & $\beta\left(K^{1 / 2}\right)$ & $E(\mathrm{~J} / \mathrm{kgmol})$ \\
\hline $\mathrm{SiH}_{4}$ & 0.3453 & 0.50 & $7.8156 \cdot 10^{7}$ \\
\hline $\begin{array}{l}\mathrm{SiH}_{2} \\
\mathrm{Si}_{2} \mathrm{H}_{6}\end{array}$ & $\begin{array}{l}6.6412 \\
4.6197\end{array}$ & $\begin{array}{l}0.50 \\
0.50\end{array}$ & $\begin{array}{l}0.0 \\
0.0\end{array}$ \\
\hline $\mathrm{H}_{2}$ & 0.0 & - & - \\
\hline $\mathrm{He}$ & 0.0 & - & - \\
\hline Ar & 0.0 & - & - \\
\hline
\end{tabular}
zero. The resultant $A, \beta$ and $E$ values are tabulated below.

\section{3 - RESULTS AND DISCUSSION}

The deposition of silicon takes place on any surface where the temperature is high enough for surface reactions to occur. Therefore, a fair comparison of Si deposition rates to experiments requires that the model keeps a correct account of the Si-carrier species inventory throughout the reactor, contrary to what most models in the literature do, i.e. to consider the susceptor or the substrate region as the only deposition surface. Thus, when calculating the si deposition rates on the susceptor top surface, the code considers the deposition on all relevant surfaces, including the quartz surface upstream of the susceptor and the vertical side and back surfaces of the susceptor.

However, the top substrate surface is the focus of this study for comparison with the experiments. Therefore, the presentation of the results will mostly concentrate on the susceptor area with the three X-slices ( $Y-Z$ plane) shown in Figure 2 at the leading edge, near the center and at the trailing edge of the susceptor.

Because of the Z-symmetry of the reactor assembly, the computational domain uses only a half of the reactor and exploits reflection boundary conditions on the central $Y-z$ plane. For the finite-difference formulation of FLUENT-CVD, we used a computational mesh for the half-reactor which had a total of 31 cells in $X, 16$ cells in $Y$ and 10 cells in $Z$ directions. The grid is nonuniformly distributed to accomodate regions of large gradients in solution variables, especially immediately above the susceptor. Even with this computational scheme, the solutions presented in this paper are still gridsensitive. However, it was ascertained that the correct physicochemical and transport phenomena are resolved. A finer mesh is not warranted with the current limitations of FLUENT-CVD and final fully converged results will be published at a later time.

This study uses the silane dissociation chemistry for silicon deposition. A 25 mole percent of either Ar (referred to as the Ar case hereafter) or He (referred to as the He case hereafter) in 75 mole percent $\mathrm{H}_{2}$ is used as the carrier gas mixture at a constant volumetric flowrate of $4 \mathrm{sim}$. These two distinct cases were chosen by Tsui because of the presumed strong buoyancydriven convection for $A r$ and the much reduced level of such convection for He. 
The added silane $(0.1$ mole percent) replaces hydrogen. Note that because the mole fractions are fixed and inert gases replace hydrogen, no accompanying changes in the chemical reaction mechanisms are expected.

Considering the couplings among the forced-convective, free-convective and diffusive transport processes in chemically reacting flow systems, choosing a criterion by which unambigious information can be extracted for the effect of only free-convection from terrestrial experiments is not trivial. Depending on the focus of interest, different experimental design conditions (such as iso-forced-convection, i.e. constant Reynolds number, iso-forced-convectiondiffusion, i.e. constant species surface flux or deposition rate when gravity is absent, iso-mass flowrate, iso-volumetric flowrate, etc.) can be employed as the basis for comparison. It should be pointed out that Tsui's experiments were run at constant total volumetric flowrates. This means that the forcedconvection. conditions (Reinolds numbers) and species diffusivities (Schmidt numbers) were different for the two $A r$ and He cases. Indeed, under the conditions of Tsui's experiments, neglecting the surface kinetic barriers, the He case is estimated to give about 13 percent higher deposition rates than the Ar case even when the buoyancy effects are absent. Therefore, the differences between the terrestrial experimental deposition rates for the Ar and He cases should be studied accordingly, i.e. if the si deposition rates (and their distribution over the substrate) are chosen to be the experimentally observable parameter to base the conclusions on, the interpretations of the deposition rate differences in the $\mathrm{Ar}-\mathrm{Ig}$ and $\mathrm{He}-1 \mathrm{~g}$ ( $\mathrm{g}$ being the terrestrial gravitational acceleration) cases need to be done with respect to the baseline $(\mathrm{Og})$ expected difference ( $\sim 13 \%$ for Tsui's experiments). Furthermore, this approach will not be able to detect the subtle self-compensating effects of buoyancy resulting from complex couplings, i.e. even when the difference between the deposition rates for the terrestrial Ar and He experiments is the same as the ones expected in the absence of gravity, one can still not conclude that the effect of buoyancy on the deposition process is negligable. This study, while assessing the type and the strength of convection in both terrestrial cases, also compares the $1 \mathrm{~g}$ cases to the respective ideal $0 \mathrm{~g}$ cases, and, hence, eliminates the ambigiuties in possible interpretations. This, of course, is one of the advantages of a computational approach.

Because of the steep temperature gradients $(2700 \mathrm{~K} / \mathrm{cm})$ and complex reactor geometry, simple dimensionless analysis using quantities such as the Grashof and the Rayleigh numbers fail to provide reliable information for the degree of significance and the specific effects of buoyancy. However, they can still be used as a guide to gain some insight for the expected magnitude of freeconvection in various cases. Consequently, the Ar-lg case is expected to have larger buoyancy effects mostly because it is heavier than He and the buoyancy effects for the He-1g case is expected to be significantly suppressed. A comparison of the fully calculated flow fields for the Ar-ig and the Ar-0g cases, performed by examining Lagrangian particle trajectories in Figure 3 , shows the dramatic 3-D influence of buoyancy. For this purpose neutralbuoyant and non-inertial particles are introduced into the stream at $0.3 \mathrm{~cm}$ above the susceptor level and their trajectories are calculated. Such numerical "flow visualization" tools clearly demonstrate the "cork-screw-like" behavior of the flow which the 2-D analyses cannot be expected to correctly describe. It should be noted that the three dimensionality of the flow inside the reactor can be observed even for the Ar-og case due to the cross-sectional flow-area changes, volumetric expansion of the gases due to thermal heating, etc.. On the other hand, a close comparison of the He-1g and He-0g cases in the present study indicates that the buoyancy effects are, indeed, significantly suppressed. It is noted that such a change is accomplished by simply replacing 25 mole percent Ar with 25 mole percent He in a 75 mole percent $\mathrm{H}_{2}$ mixture. The subtle differences between the He-1g and $\mathrm{He}-\mathrm{Og}$ cases and how they may be important in some CVD applications are beyond the scope of the objectives of this paper and will be elaborated upon elsewhere (Gokoglu et al., to be published). Therefore, we will omit the presentation of the He-Og cases in this specific study and point out, again, that the overall flow features in both cases result in similar transport phenomena, and consequently, similar Si deposition rates. 
Figure 4 shows the velocity vectors at the previously designated three $\mathrm{X}$-slices for the three cases: $\mathrm{Ar}-1 \mathrm{~g}, \mathrm{Ar}-0 \mathrm{~g}$ and He-1g. The complex flow pattern for Ar-1g should be contrasted with the other two and, especially, with the smooth behavior of Ar-Og where there are no vectors pointing opposite to the main flow direction (reverse flow). The compaxison of Ar-1g and He-ig demonstrates the intensity of buoyancy-driven convection rolls for Ar-1g and how it influences the flow on the susceptor. In fact, it has been computationally determined, although not readily observable from Figure 4, that. the number of free-convection cells across the width of the reactor varies along the susceptor length. The presence of the vertical drops on both sides of the susceptor helps to stabilize the flow on the susceptor, as is evident from the He-1g case, by localizing the gravitationally-induced rolls to the drop region. The He-og case (not shown) is qualitatively like Ar-Og with no reverse flows or free-convection cells and, therefore, is fundamentally different from the He-1g case. However, as is mentioned above, buoyancy effects are so substantially supressed for He-1g in this study that the presentation of He-0g results is found to be redundant with respect to the He-ig case.

Figure 5 shows $z$-slices of the modelled reactor volume for velocity, temperature and silane concentration fields. Except for cases (b) and (c), these slices are taken $0.19 \mathrm{~cm}$ away from the center symmetry-plane. For cases (b) and (c), however, they are taken $2.10 \mathrm{~cm}$ away from the center symmetryplane and are slightly beyond the susceptor. The cases (a), (b) and (c) depict the existance of a convection cell also in the longitudinal direction, which is stronger for Ar-1g than for He-1g. Case (a) shows that the reversed flow in the lower portion of the reactor exit zone rotates towards the top of the reactor and significantly affects the axial main flow at the trailing edge of the susceptor. Case (b) shows that the reversed flow extends further upstream along the sides of the susceptor, turn up and intensify due to the heated susceptor side walls, and, eventually, turn around towards the reactor exit. It is interesting to observe that while the flow naturally "falls" after every geometric down-step for $0 \mathrm{~g}$ cases, as one would intuitively expect, it actually rises for $1 \mathrm{~g}$ cases. The presence of these longitudinal cells on both sides of the susceptor implies, especially for Ar-1g, that the main axial flow on the susceptor is likely squeezed from both sides and accelerates through the reduced effective flow-area. Case (c) demonstrates the apparent supression of this buoyancy related phenomenon by helium.

Cases (d), (e) and (f) in Figure 5 are isotherm contour plots for Ar-0g, Ar-1g and He-1g displaying also the calculated heating of the surface upstream of the susceptor and the temperature distribution inside the solid bodies. Note that the susceptor is kept at a constant temperature of $1373 \mathrm{~K}$. The comparison of (d) and (e) shows how the temperature field is modified by the freeconvection effects, whereas the comparison of (e) and ( $f$ ) shows the relative effect of different Prandtl numbers (the ratio of the kinematic viscosity, $\mu / \rho$, to the thermal diffusivity, $\lambda /\left(\rho c_{p}\right)$, of the gas mixture) on the temperature distribution.

Cases ( $g$ ), ( $h$ ) and ( $i$ ) display the relative effects of convection and diffusion on silane depletion (related to Si deposition) rates. The presence of free-convection facilitates the convective mass transport to the deposition surface resulting in more depletion of the source gas silane. However, because the diffusional mass transport is very significant for the delivery of Si-carrier species to the surface in this study, using a He host gas mixture depletes more silane even with supressed free-convection.

Figures 6,7 and 8 are 2-D contour plots of temperature and silane, silylene and disilane concentrations around the susceptor at the three X-sliced locations along the susceptor for the Ar-1g, Ar-Og and He-1g cases, respectively. The isotherms in Figure 6 demonstrate clearly how freeconvection distorts the temperature profiles around the susceptor for Ar-lg. As the thermal field develops and the number of free-convection cells varies over the susceptor along the flow axis, the nature of the distortion also varies, both qualitatively and quantitatively. In fact, for Ar-1g the thermal 
Field never becomes fully developed over the susceptor in this particular study, although for $\mathrm{Ar}-\mathrm{Og}$ and $\mathrm{He}-\mathrm{Ig}$ it does. The consequent appearance of the species concentration fields is influenced in two major ways due to these buoyancy effects. Firstly, the rates of homogeneous reactions that produce the reactive species respond exponentialiy to the local temperature; and, secondly., the free-convective transport of species both in the $Y-Z$ planes (cross-sectional cells) and in the X-Y planes (longitudinal cells) affects both the local homogeneous reaction and transport rates. Note that when the buoyancy effects are eliminated or supressed (Figures 7 and 8 ) the problem can be analyzed fairly accurately by (pseudo)2-D treatments which consider the flow and thermal field developments and their subsequent effects on the concentration fields using boundary layer approximations. In such cases, cross-sectional and longitudinal mixing will depend basically on diffusion.

A comparison of $\mathrm{SiH}_{4}$ concentration contours among different $\mathrm{X}$-slices in Figures $6(\mathrm{~b}), 7(\mathrm{~b})$ and $8(\mathrm{~b})$ indicates that the concentration of silane gets smaller closer to the hot substrate surface. This is attributed to the formation and faster surface reaction (deposition) of the other reactive species, $\mathrm{SiH}_{2}$ and $\mathrm{Si}_{2} \mathrm{H}_{6}$, rather than to the relatively much slower surface reaction (deposition) of $\mathrm{SiH}_{4}$ at the susceptor temperature of this study. Indeed, this assertion is confirmed by the further analysis of numerical data which indicates that, among the three si-carrier species, the contribution of the $\mathrm{SiH}_{4}$ species to the total $\mathrm{Si}$ deposition rate is negligably small as compared to the other two. As can be seen from the comparison of Figures $6(b)$ and $7(b)$, the depletion of silane is more for Ar-1g than for Ar-Og due to the enhanced transport of the source to the reaction zones by free-convection. In fact, the uniform cross-sectional silane concentration observed at the reactor exit-plane for $\mathrm{Ig}$ cases is the result of such more efficient mixing, although a silane concentration gradient still exists across the reactor exit-plane for og cases.

The dissociation reactions for $\mathrm{SiH}_{4}$ and $\mathrm{Si}_{2} \mathrm{H}_{6}$ are favored at higher temperatures. Therefore, the formation of $\mathrm{SiH}_{2}$ is promoted closer to the hot substrate surface. It is observed, although not readily seen from Figures $6(\mathrm{c}), 7(\mathrm{c})$ and $8(\mathrm{c})$ due to the insufficient resolution, that $\mathrm{SiH}_{2}$ has a concentration peak close to the hot surface. It gets less concentrated in the cooler regions due to slower $\mathrm{SiH}_{4}$ dissociation and faster $\mathrm{Si}_{2} \mathrm{H}_{6}$ formation, and less concentrated in the immediate vicinity of the hot surface due to the instantaneous surface reaction, resulting in positive diffusional flux toward the surface.

The formation of $\mathrm{Si}_{2} \mathrm{H}_{6}$, Figures $6(\mathrm{~d}), 7(\mathrm{~d})$ and $8(\mathrm{~d})$, in cooler regions for $1 \mathrm{~g}$ cases is promoted by the free-convection facilitated transport of $\mathrm{SiH}_{2}$, which has been formed in hotter regions. The reader is alerted to the fact that $\mathrm{Si}_{2} \mathrm{H}_{6}$ contour values are one to two orders of magnitude smaller than $\mathrm{SiH}_{2}$ values. Concentration contours for $\mathrm{Si}_{2} \mathrm{H}_{6}$ at the susceptor leading edge show that, similar to $\mathrm{SiH}_{2}$, a maximum exists also for $\mathrm{Si}_{2} \mathrm{H}_{6}$. However, subsequent $\mathrm{X}$-slices downstream of the susceptor show that $\mathrm{Si}_{2} \mathrm{H}_{6}$ concentration uniformly decays toward the susceptor top surface, suggesting that the homogeneous reactions reach equilibrium and the $\mathrm{Si}_{2} \mathrm{H}_{6}$ concentration field is governed by the transport rates with an infinately fast surface reaction (relative to transport rates). It should be noted that although the gas phase chemical reaction mechanisms are the same for both Ar and He cases, the significance of gas phase reactions are different for each case depending on the relative rates of convective and diffusive transport.

Figure 9 depicts the contour plots of resulting $S i$ deposition rates obtained from our computations in comparison to 'Tsui's/16/ corresponding experiments. It should be mentioned that the experimental contours are generated from a total of only twelve data points for illustrative comparison purposes. The measurements were made at the intersections of four equally-spaced rows and three equally-spaced columns outlined in the central region of the susceptor. Therefore, the shapes of the experimental contours should be interpreted accordingly. All of the three calculated deposition rate contours show the same trend of decreasing rates toward the center and trailing edge of the susceptor, resembling an amphitheater. Regardless of the level of free- 
convection, these shapes reflect a) the depletion of S1-carrier source species by deposition toward the trailing edge, resulting in lower rates, and b) the steeper gradients of solution variables toward the sides due to the geometric and thermal setup, resulting in higher rates. The widthwise variation of deposition rates is affected by the cross-sectional free-convection cells in two ways : 1). the rates tend to be further reduced at the center symmetryplane and enhanced at the sides because the cells rotate up toward the top wall of the reactor at the center and down along the sides, and 2) the rates tend to be augmented, though unevenly, everywhere along the width by the facilitated supply of reactant species from higher concentration regions to deprived areas. These two competing effects result in the overall enhancement of rates, Figure $9(\mathrm{a})$ and (c), with varying magnitudes along both the width and length of the susceptor.

A comparison of integrated deposition rates over the total surface of the susceptor for Ar-1g, He-1g and $\mathrm{Ar}-0 \mathrm{~g}$, Figure $9(\mathrm{a})-(\mathrm{C})$, indicate that the highest rate is obtained for He-1g and the lowest rate is for. Ar-0g.

Considering that free-convection is significantly suppressed for He-1g, this shows that the faster diffusion rates of si-carrier species in He (versus in Ar) has a more pronounced effect in enhancing rates than free-convection.

A comparison of computational and experimental deposition rate results, Figure 9 (a) and (b), reveals that our preliminary modelling effort is capable of predicting the qualitative and quantitative behavior of $\mathrm{si}$ deposition from silane with a remarkable accuracy. Although the axial trends are more correctly predicted, the difference in widthwise variation can partially be attributed to the scarcity of experimental data.

\section{4 - CONCLUSIONS AND CLOSING REMARRS}

The computational fluid dynamics code FLUENT-CVD has been adopted to simulate a 3-D experimental CVD reactor. The code incorporates the effects of both homogeneous (gas phase) and heterogeneous (surface) chemistry. It has been applied to silicon deposition from silane at a substrate temperature of $1373 \mathrm{~K}$ using the finite rate homogeneous reactions relevant to silylene and disilane. Either a $25 \%$ helium or a $25 \%$ argon by mole with hydrogen balance is used as the host gas mixture for comparison with experiments. These two cases were shown to have appreciable (Ar mixture) or supressed (He mixture) leveis of gravitationally-induced free-convection. The simulations were also carried out in the absence of gravity to eliminate free-convection. The complex 3-D nature of the flow under the terrestrial gravitational field has been demonstrated. The reported results are considered to be preliminary due to the current limitations of the code and the relatively crude computational mesh used. A more thorough analysis will be published later. However, the correct physicoand thermo-chemical phenomena have been sufficiently resolved in the present study. The numerical results compare favorably with experiments.

For the case presented here, a major enhancement of the code will be the inclusion of thermal (Soret) diffusion. A more meaningful code verification study with a finer computational mesh can then be carried out using Tsui's/16/ data for various $\mathrm{Ar}-\mathrm{H}_{2}$ and $\mathrm{He}-\mathrm{H}_{2}$ mixture ratios at different substrate temperatures, in the absence of homogeneous nucleation. The capabilities of the code can be assessed for the extreme limits where there are no homogeneous and/or heterogeneous chemical reaction barriers. The effects of changes in the reactor geometry, orientation with respect to the gravity vector, uncertainties in chemical kinetic parameters, etc. will be the subject of our future publications.

The improved design and optimization of future CVD reactors requires improved and optimized computer codes. It is essential that the data fed to such computational codes be of utmost quality. However, the refinement of such input information can more efficiently be achieved via more focused experimental and theoretical efforts. Therefore, modelling efforts addressing 
specific aspects of a complex phenomena in a better defined environment/24/, or smaller scale experimental efforts geared towards providing answers to individual thermo-chemical questions related to CVD are integral parts of a coordinated program. Only when such a loop is closed can computationai codes go beyond research purposes providing invaluable insights and be confidently used as powerful design tools.

Acknowledgements : It is a pleasure to acknowledge the computational assistance of Mr. Ron Gaug (Univ. of Akron, Akron, Ohio). Special thanks are also due to Mr. Kurt Fretz, Drs. Tom Jasinski and Zahed Sheikholeslami (Creare, Inc., Hanover, New Hampshire) for their help with FLUENT-CVD.

\section{LIST OF SYMBOLS}

A pre-exponential factor for Arrhenius reaction rate expression.

${ }_{D}^{C}$ Fick diffusion coefficient.

E activation energy for Arrhenius reaction rate expression.

$g$ earth gravitational acceleration.

h enthalpy of gas mixture.

$\mathrm{J}$ total number of gas phase species.

$k$ reaction rate constant.

$\mathrm{K}$ total number of reactions.

n unit vector normal to surface.

p pressure.

$R$ universal gas constant.

$R_{j k}$ mass rate of creation of species $j$ per unit volume (gas phase)

$s$ by reaction $k$.

$R_{j k}^{S}$ mass rate of creation of species $j$ per unit area (surface) by reaction $k$.

Sc Schmidt number, $\mu /(\rho D)$.

$T$ temperature in Kelvin degrees.

u velocity.

W molecular weight.

$\mathrm{x}$ dimension coordinate.

$Y$ mass fraction.

$\beta \quad$ exponent of $T$ in Arrhenius reaction rate expession.

$\gamma \quad$ surface reaction probability.

$\lambda$ thermal conductivity of gas mixture.

$\mu$ dynamic viscosity of gas mixture.

$\rho$ density of gas mixture.

a partial differential operator.

$\nabla$ vector differential operator.

\section{Subscripts}

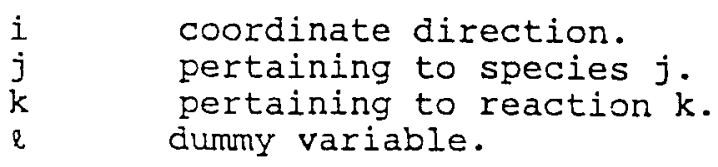

\section{Superscripts}

$\mathrm{s}$

pertaining to surface. 


\section{REFERENCES}

/1/ Hess, D. W., Jensen, K. F. and Anderson, T. J., Rev. Chem. Eng. 3 (1985) 97.

/2/ Jensen, K. F., Chem. Eng. Sci. 42 (i987) 923.

13/ Rosenberger, $F$, Chemical Vapor Deposition 1987, Electrochem. Soc., Ed. G. W. CuIlen (1987) 11 .

/4/ Rebenne, H. and Pollard, R., J. Am. Ceram. Soc. 70 (1987) 907.

/5/ Houfman, C., Graves, D. B. and Jensen, K. F., J. Electrochem. Soc. 133 (1986) 961 .

16./ Wahl, G., Schmaderer, F., Huber, R. and Weber, R., Chemical Vapor Deposition 1987, Electrochem. Soc., Ed. G. W. Culien (1987) 42 .

/7/ Evans,, G. and Greif, R., Proc. 1987 ASME-JSME Thermal Engineering Joint Conf., Vol. 1 (1987) 589 .

/8/ Coltrin, M. E., Kee, R. J., Evans, G. H. and Miller, J. A., Chemical Vapor Deposition 1987, Electrochem. Soc., Ed. G. W. Cullen (1987) 33.

19/ Coltrin, M. E., Kee, R. J. and Miller, J. A., J. Electrochem. Soc. 131 (1984) 425 .

/10/ Coltrin,.M. E., Kee, R.J. and Miller, J. A., J. Electrochem. Soc. 133 $(1986) 1206$.

/11/ Ristorcelli, J. R.Jr. and Mahajan, R. L., Proc. 1987 ASME-JSME Thermal Engineering Joint Conf., Vol. 1 (1987) 613.

/12/ Rhee, S., SzekelY, J. and Ilegbusi, O. J., J. Electrochem. Soc. 134 (1987) 2552 .

/13/ Moffat, H. K. and Jensen, K. F., J. Electrochem. Soc. 135 (1988) 459.

/14/ Jasinski, T., Sheikholeslami, M. Z. and Fretz, K. W., AIChE Symposium Series, Ed. S. B. Yilmaz, Vol. 84, No. 263 (1988) 79.

/15/ Sheikholeslami, M. 2., Jasinski, T. and Fretz, K. W., AIAA First National Fluid Dynamics Congress, Cincinnati OH (1988).

116/ Tsui, P., Penn. State Univ., Dept. of Materials science and Engineering, Ph. D. Thesis (1988).

/17/ Eversteyn, F. C., Severin, P. J. W., van den Brekel, C. H. J. and Peek, H. L., J. Electrochem. Soc. 117 (1970) 925.

118/ Stinespring, C. D. and Annen, K. D., AIAA-87-0313 (1987).

/19/ "FLUENT MANUAL", TN-369, Rev. 3, Creare Inc., Hanover, NH, February (1987).

/20/ Jasinski, T., Sheikholeslami, M. Z. and Fretz, K. W., Phase I Final Report, NASA: Contract NASI-18413, Creare Inc., Hanover,.. NH, TM-11.78 (1987).

/21/ Bird, R. 'B.', Steward, W. E. and Lightfoot, E. N., Transport Phenomena', John Wiley \& Sons, New York (1960) 258.

/22/ Rosner, D. E., Physicochem. Hydrodyn. i (1980) 159.

/23/ Quazzani, J., Chiu, K. -C. and Rosenberger, E., Chemical vapor Deposition 1987, Electrochem. Soc., Ed. C. W. Cullen (1987) 165.

/24/ Gokoglu, S. A., J. Electrochem. Soc. 135 (1988) 1562 .

TABLE I : Contour values defined in Figs. 5-8

\begin{tabular}{|c|c|c|c|c|}
\hline Contour & Temp. , $\mathrm{K}$ & $\mathrm{SiH}_{4}$ & $\begin{array}{l}\text { S FRACTION } \\
\mathrm{SiH}_{2}\end{array}$ & $\mathrm{Si}_{2} \mathrm{H}_{6}$ \\
\hline A & $1.33(+3)$ & $1.2(-2)$ & $3.4(-4)$ & $2.0(-5)$ \\
\hline B & $1.06(+3)$ & $9.0(-3)$ & $2.8(-4)$ & $1.1(-5)$ \\
\hline C & $7.92(+2)$ & $5.8(-3)$ & $2.2(-4)$ & $4.0(-6)$ \\
\hline D & $5.24(+2)$ & $2.7(-3)$ & $1.6(-4)$ & $3.1(-6)$ \\
\hline$E$ & - & $1.8 i-3)$ & $1.4(-4)$ & $1.0(-6)$ \\
\hline $\mathrm{F}$ & -- & $1.1(-3)$ & $1.0(-4)$ & $2.0(-7)$ \\
\hline G & -- & $3.5(-4)$ & $2.0(-5)$ & -- \\
\hline $\mathrm{H}$ & -- & -- & $1.5(-5)$ & -- \\
\hline
\end{tabular}




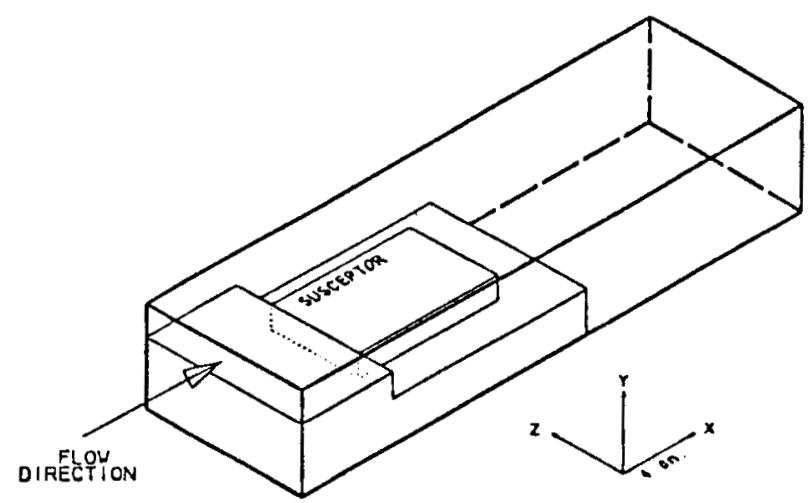

Figure 1.

The schematic of the modelled portion of the reactor.

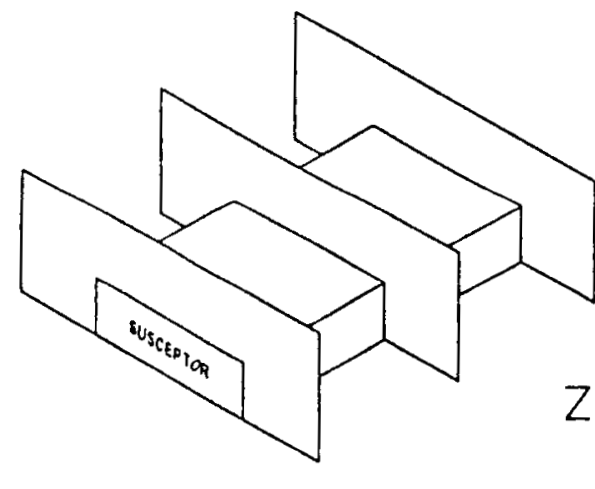

Figure 2.

Magnified view of the susceptor section, showing three $\mathrm{x}$-slices at $0.0 \mathrm{~cm}, 3.58 \mathrm{~cm}$ and $7.0 \mathrm{~cm}$ from the susceptor leading edge. Data in Figures 4 and $6-9$ are presented according to this format.
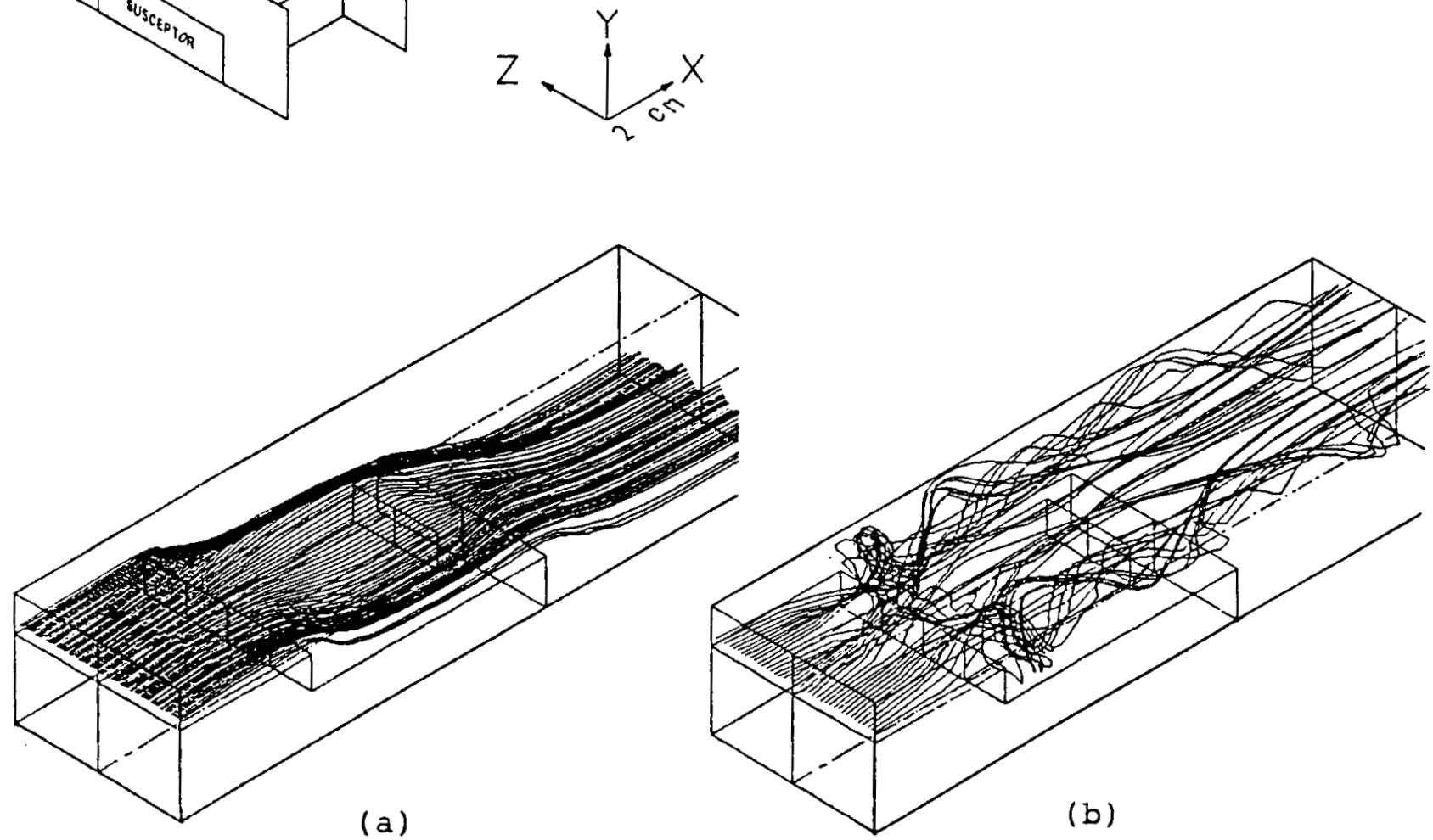

Figure 3.

Trajectories of particles introduced $0.3 \mathrm{~cm}$ above the surface at the entrance of the modelled reactor volume for (a) Ar-0g, and (b) Ar-1g. 


\section{ORIGINAL PAGE IS
OF POOR QUALITY}

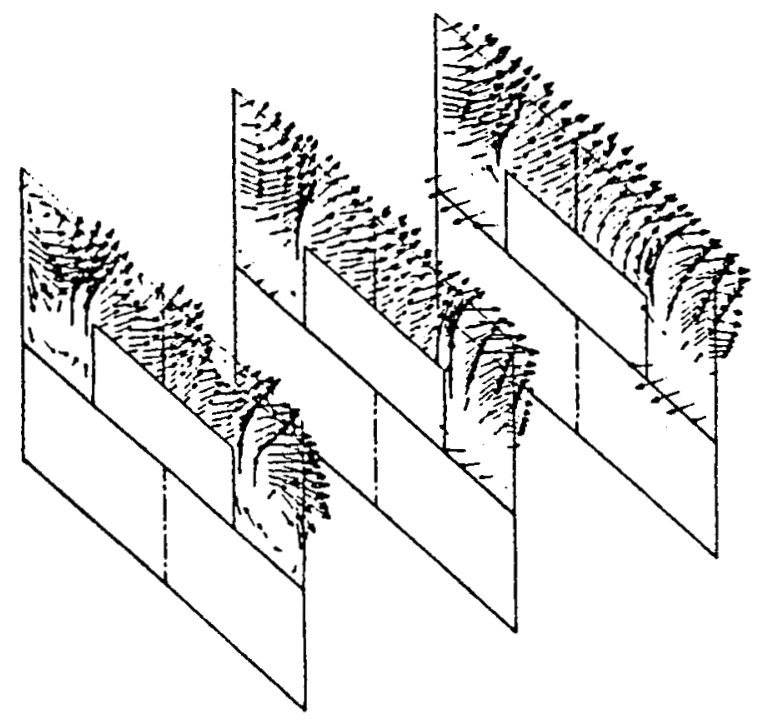

(a) Max.Vel. $=2.47(-1) \mathrm{m} / \mathrm{s}$

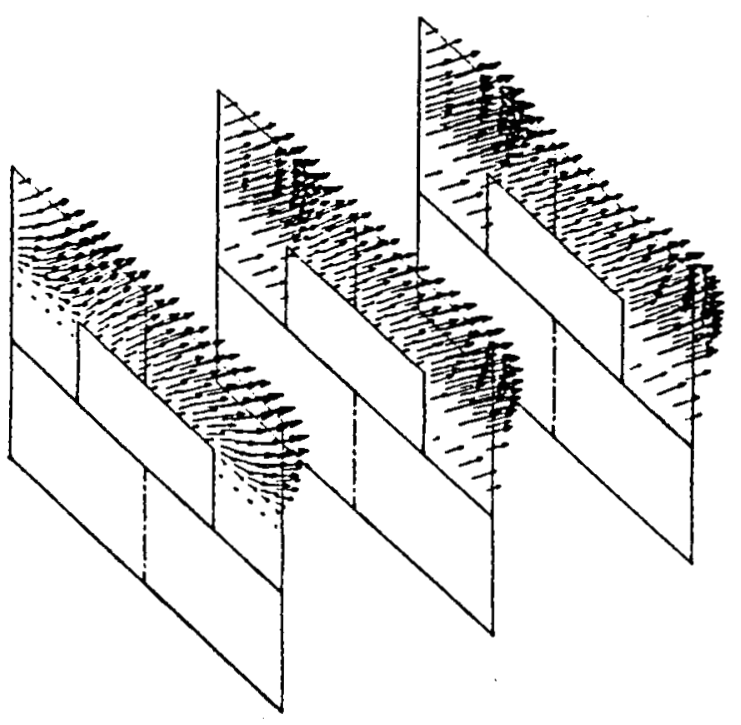

(b) Max.Vel. $=1.37(-1) \mathrm{m} / \mathrm{s}$

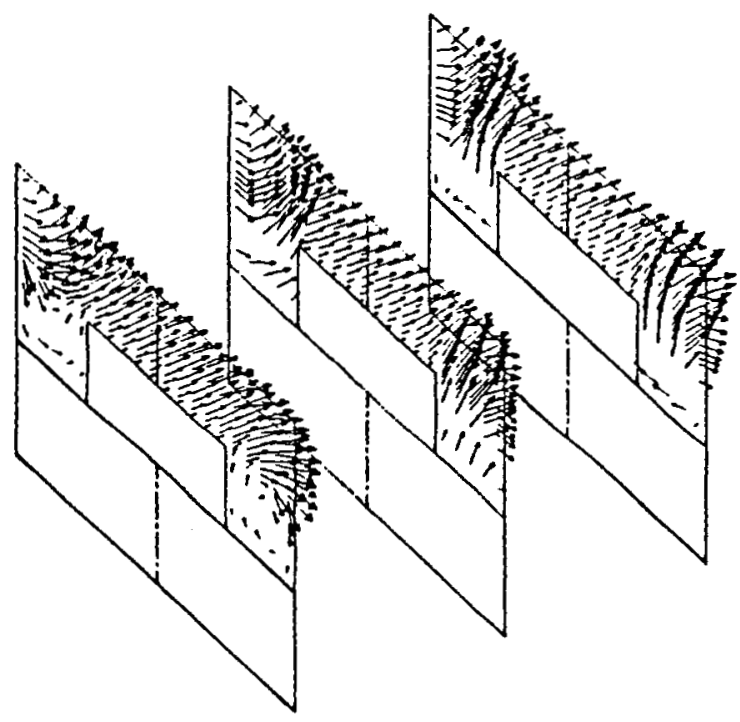

(c) Max.Vel. $=2.33(-1) \mathrm{m} / \mathrm{s}$

Figure 4.

The velocity vectors at the three $\mathrm{x}$-slices for (a) Ar-1g, (b) Ar-Og, and (c) He-1g. Magnitude of vectors is proportional to the maximum velocity stated. 


\section{ORIGINAL PAGE IS \\ OF. POOR QUALITY}
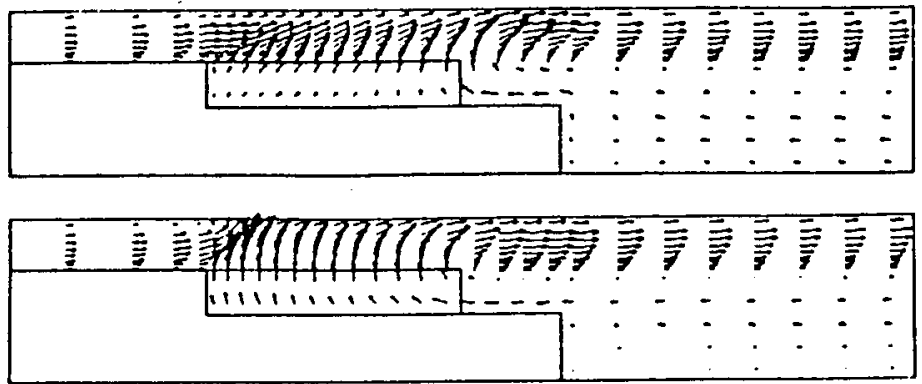
(b) Ar-1g
2.10

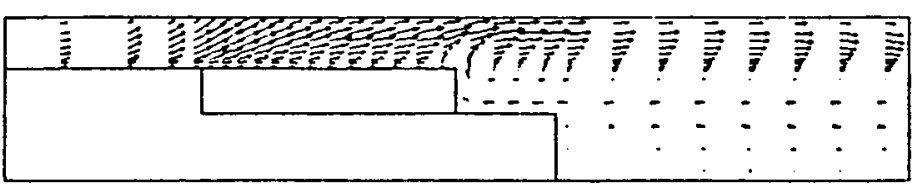

(c) $\mathrm{He}-1 \mathrm{~g}$

2.10
Position from center symmetry plane $(\mathrm{cm})$

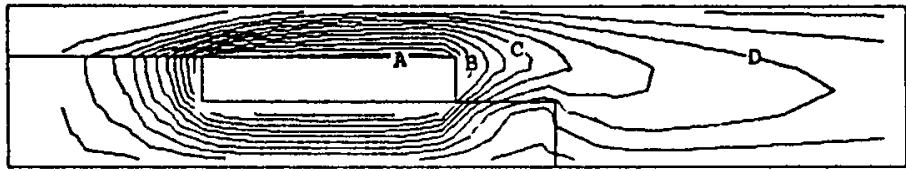
(d) $\mathrm{Ar}-\mathrm{Og}$
0.19

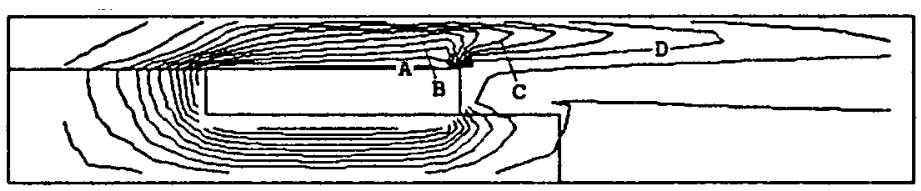
(e) Ar-1g
0.19

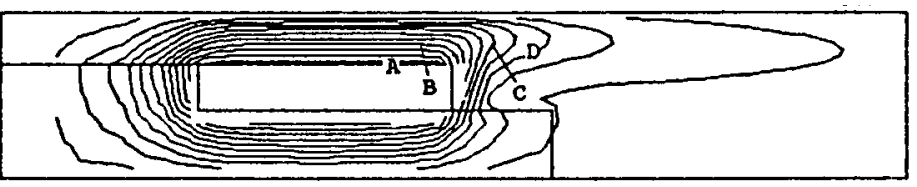

(f) $\mathrm{He}-1 \mathrm{~g} \quad 0.19$

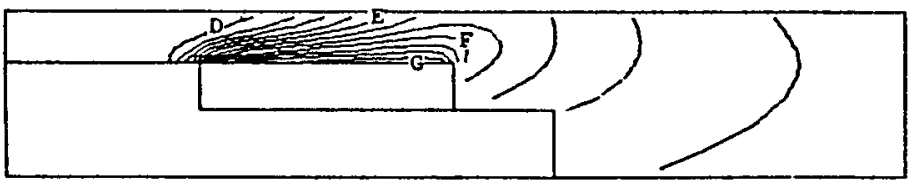
(g) Ar-Og
0.19

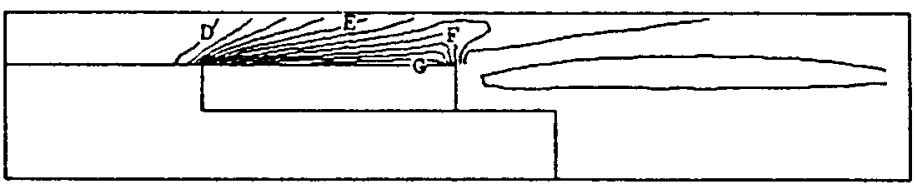
(h) Ar-1g
0.19

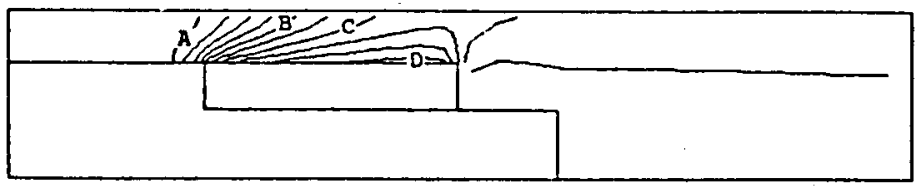

(i) $\mathrm{He}-1 \mathrm{~g} \quad 0.19$

Figure 5.

Z-sliced velocity vectors $(a)-(c)$, isotherms $(d)-(f)$, and silane concentration contours $(g)-(i)$. Slice locations and cases considered are as marked. Refer to Table I for contour values. 


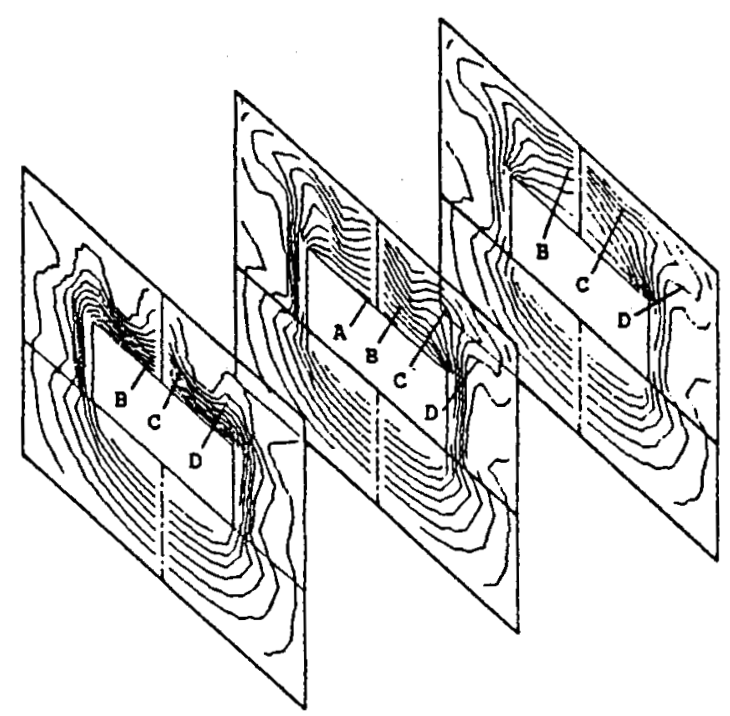

(a)

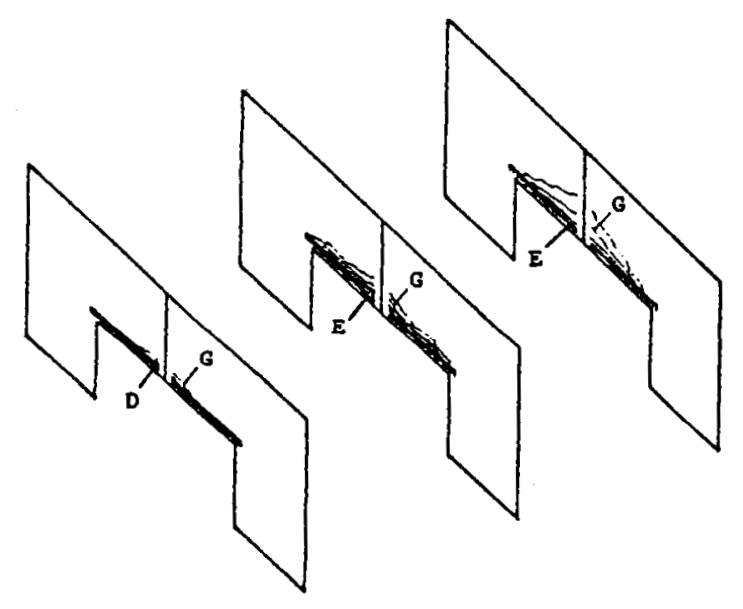

(c)

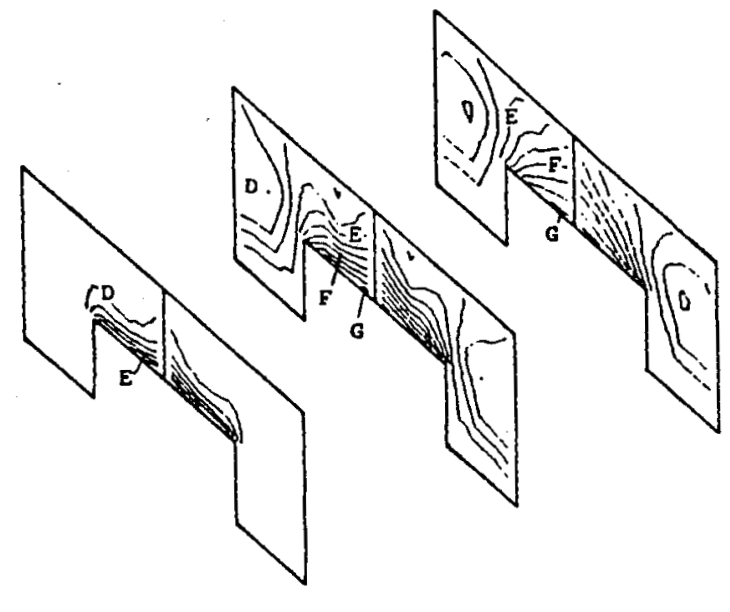

(b)

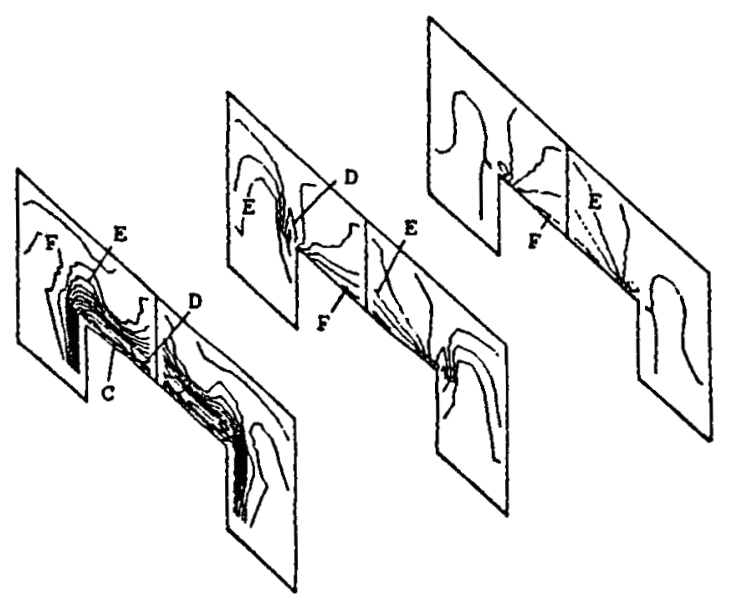

(d)

Figure 6 .

The $x-s l i c e s$ for Ar-1g: (a) isotherms, and concentration contours for (b) $\mathrm{SiH}_{4}$, (c) $\mathrm{SiH}_{2}$ and (d) $\mathrm{Si}_{2} \mathrm{H}_{6}$. Refer to Table I for contour values. 

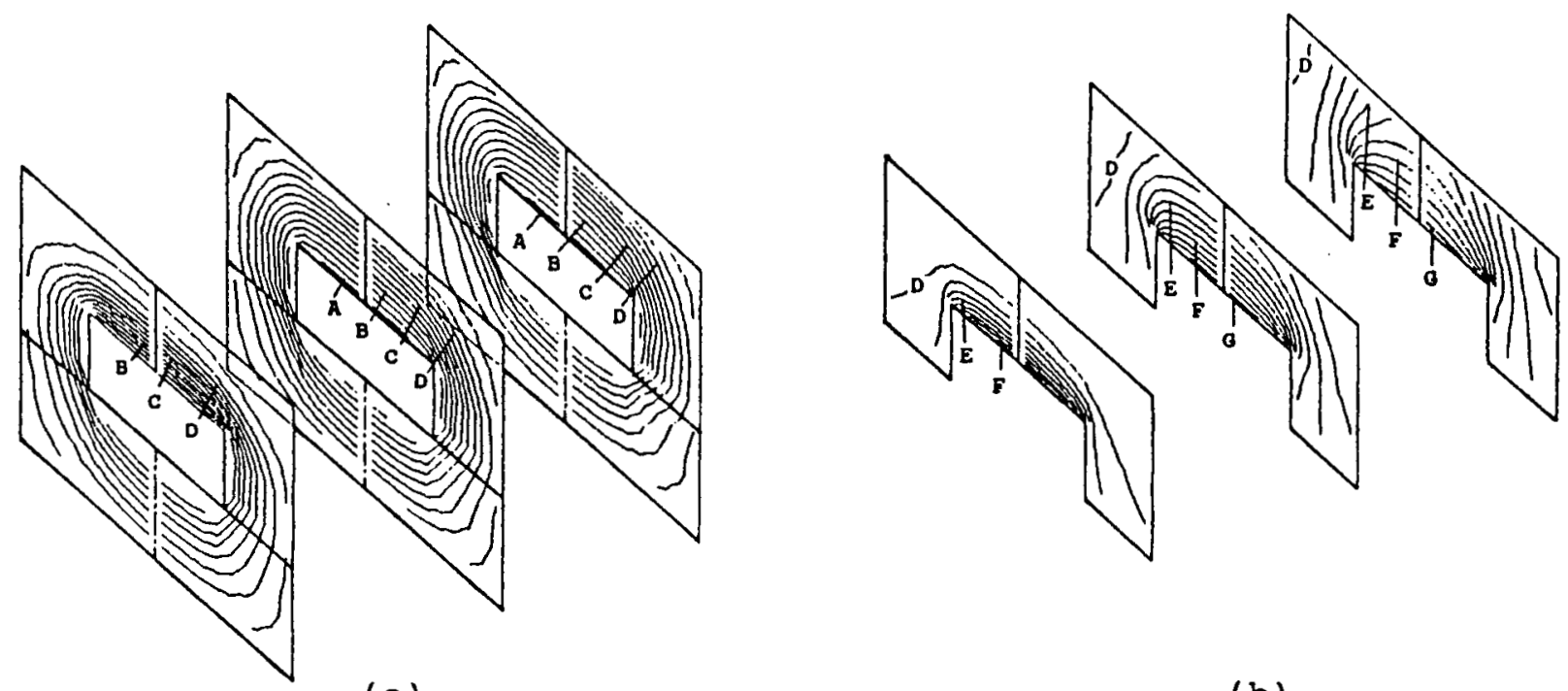

(a)

(b)

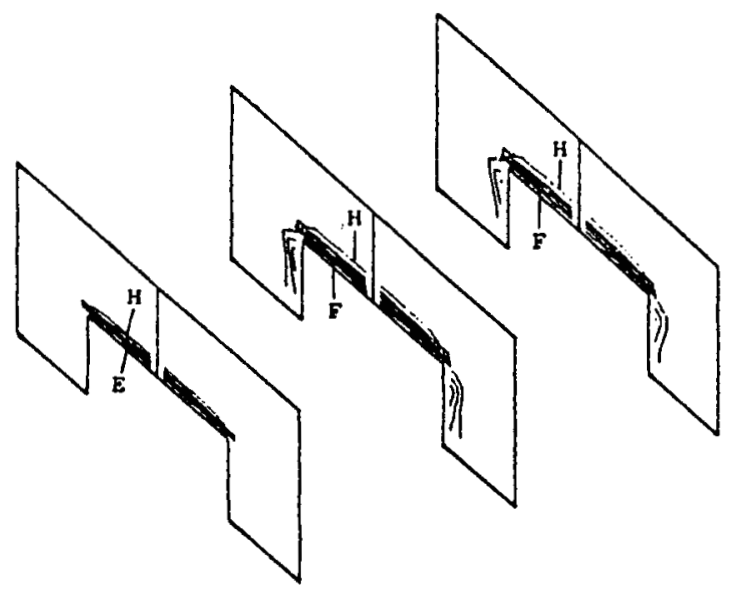

(c)

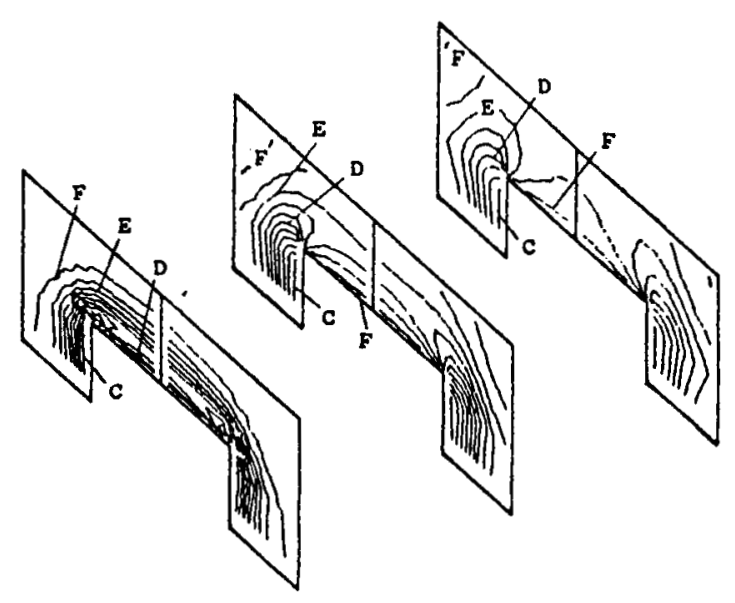

(d)

Figure 7.

The $x$-slices for Ar-Og: (a) isotherms, and concentration contours for (b) $\mathrm{SiH}_{4}$, (c) $\mathrm{SiH}_{2}$ and (d) $\mathrm{Si}_{2} \mathrm{H}_{6}$. Refer to Table I for contour values. 


\section{ORIGINAL FAGE IS \\ OF POOR QUALITY}
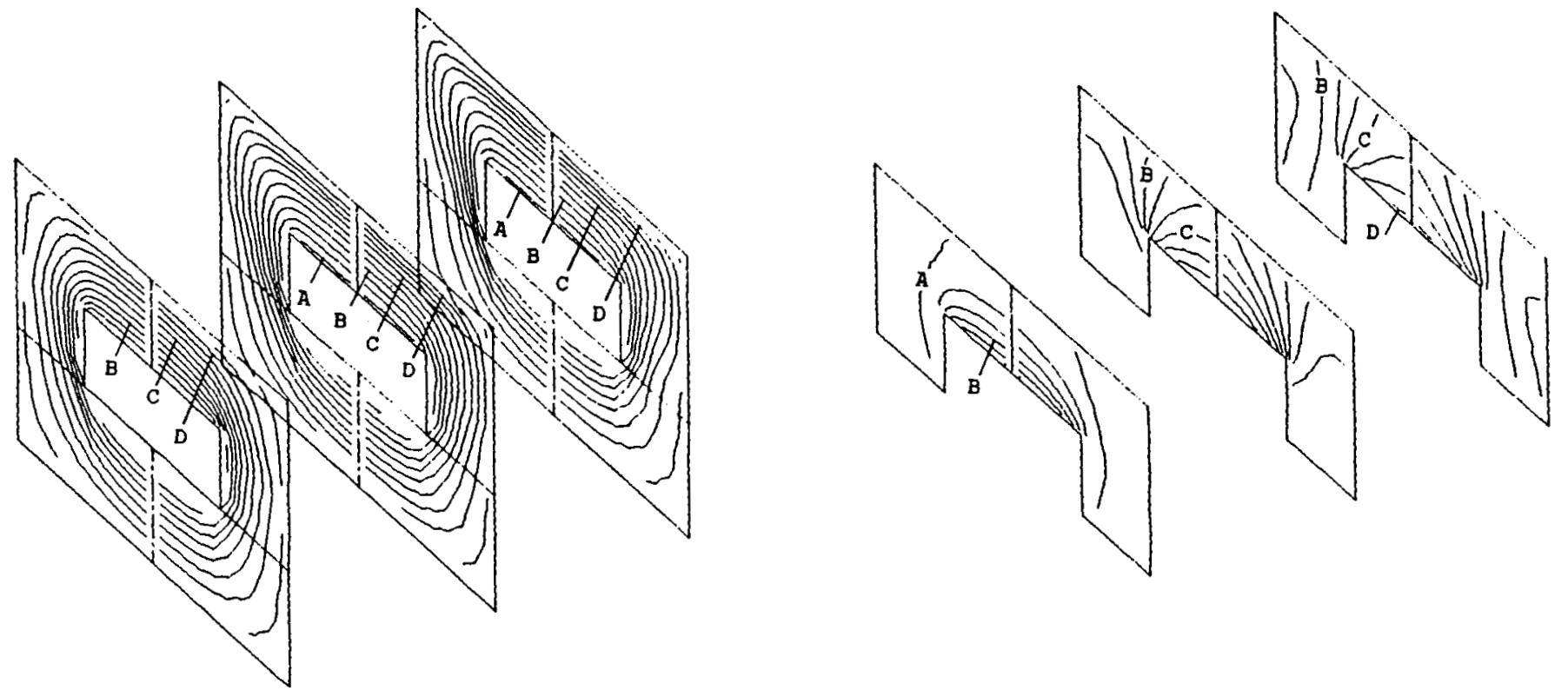

(a)

(b)

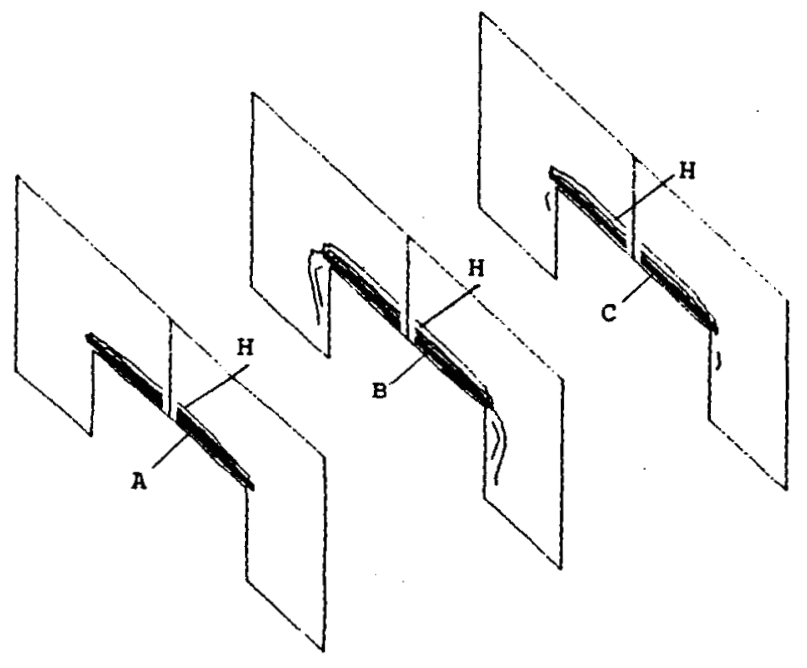

(c)

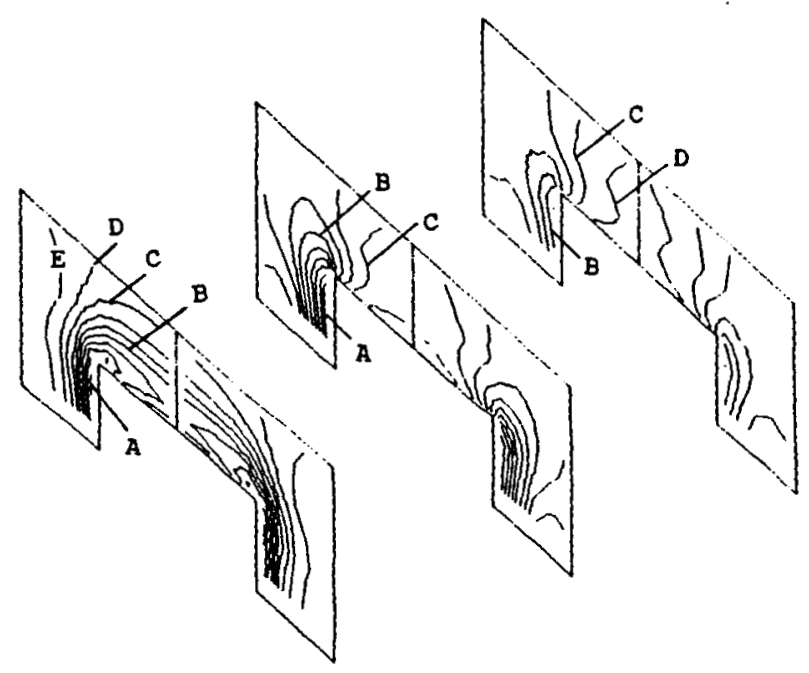

(d)

Figure 8 .

The $x-s l i c e s$ for He-1g: (a) isothems, and concentration contours for (b) $\mathrm{SiH}_{4}$, (c) $\mathrm{SiH}$ and (d) $\mathrm{Si}_{\mathbf{3}} \mathrm{H}_{6}$. Refer to Table I for contour values. 
느은 느는 눙

NNmm何

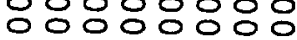

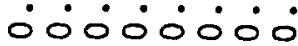

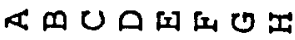

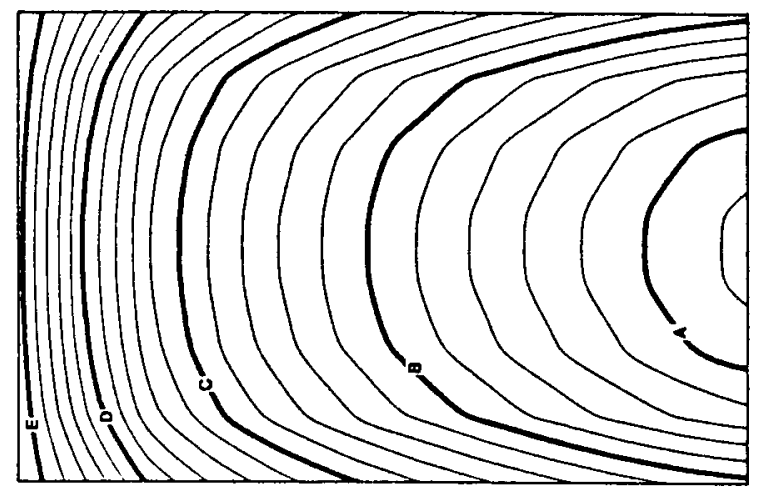

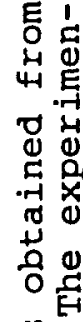

u

学

0 O

म

ज断

E

过第

H

我 50

둥ㅇ

○云起

봉

E

兵

4.

4 मै है

$\rightarrow$ م.न-

थ

4 号

0 需

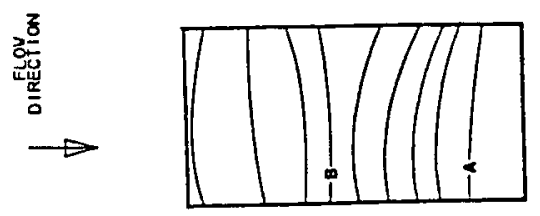

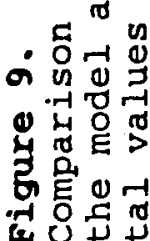

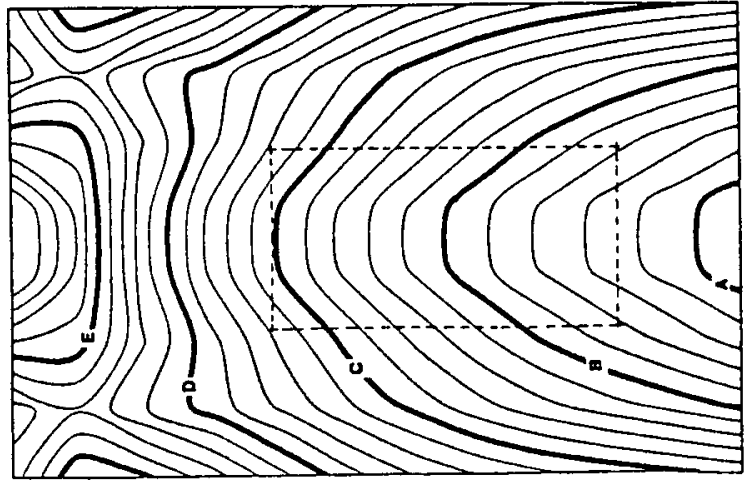




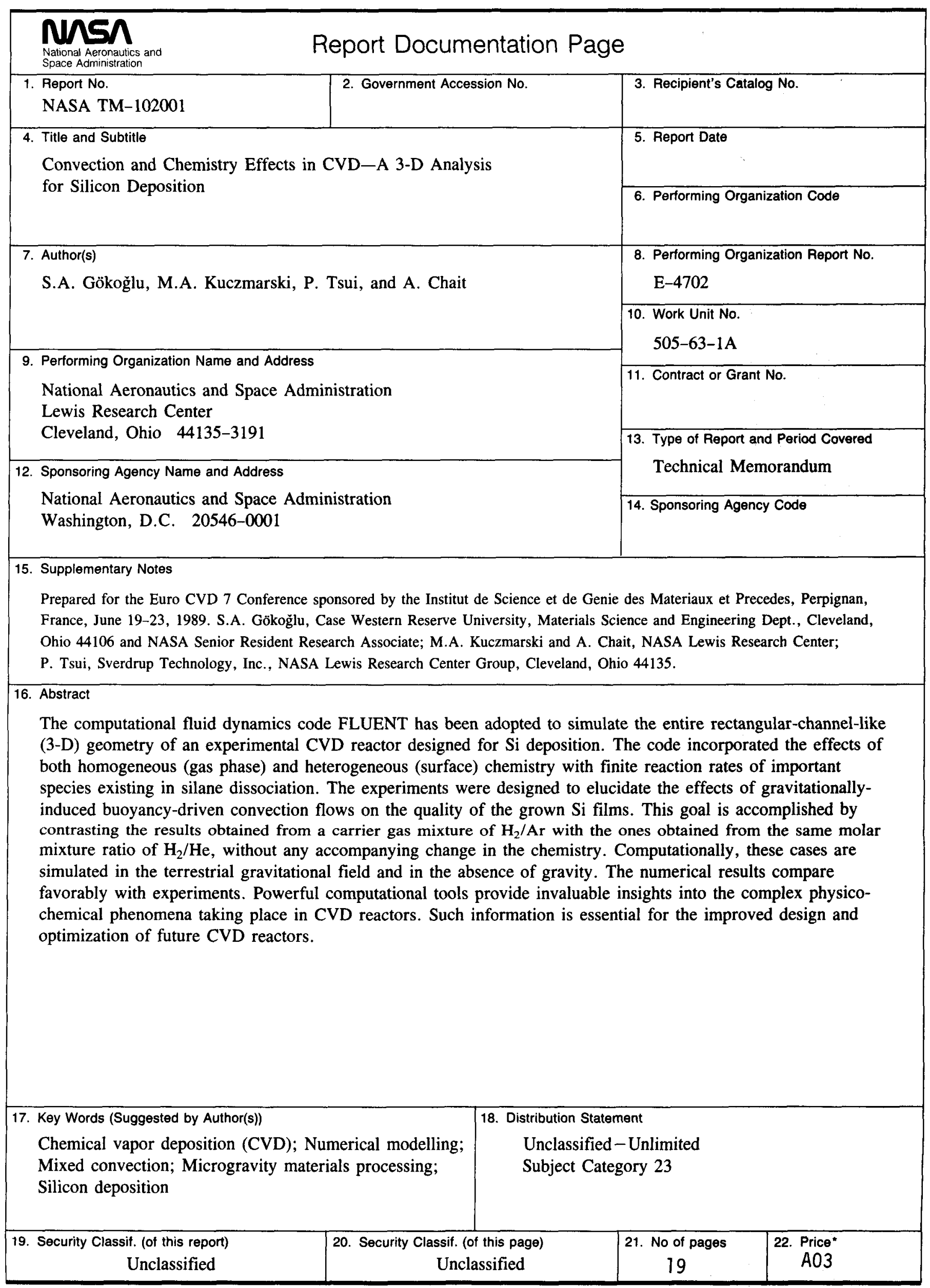

